(2) Open Access Full Text Article

\title{
Crosstalk between EGFR and integrin affects invasion and proliferation of gastric cancer cell line, SGC790 I
}

This article was published in the following Dove Press journal:

OncoTargets and Therapy

16 October 2012

Number of times this article has been viewed

Li Dan ${ }^{1, *}$

Ding Jian ${ }^{2, *}$

Lin $\mathrm{Na}^{\prime}$

Wang Xiaozhong'

'Digestive Department, the Union Hospital of Fujian Medical University, Fujian, People's Republic of China; ${ }^{2}$ Digestive Department, the First Affiliated Hospital of Fujian Medical University, Fuzhou, Fujian, People's Republic of China

*These authors contributed equally to this work
Correspondence: Ding Jian

Digestive Department, the First Affiliated Hospital of Fujian Medical University, Chating Street, 350002, Fuzhou, Fujian, People's Republic of China Email docdingjian@yahoo.com.cn
Background/objective: To investigate the crosstalk between epidermal growth factor receptor (EGFR) and integrin-mediated signal transduction pathways in human gastric adenocarcinoma cells.

Methods: EGF was used as a ligand of EGFR to stimulate the gastric adenocarcinoma cell, SGC7901. Signal molecules downstream of the integrin, FAK(Y397) and p130cas(Y410) phosphorylation, were measured by immunoprecipitation and western blot. Fibronectin (Fn) was used as a ligand of integrin to stimulate the same cell line. Signal molecules downstream of EGFR and extracellular signal-regulated kinase (ERK) general phosphorylation were also measured. Focal adhesion kinase (FAK) small-interfering RNA was designed and transfected into SGC7901 cells to decrease the expression of FAK. Modified Boyden chambers and MTT assay were used to examine the effect of FAK inhibition on the invasiveness and proliferation of SGC7901.

Results: EGF activated FAK(Y397) and p130cas(Y410) phosphorylation, while Fn activated ERK general phosphorylation. Inhibition of FAK expression decreased p130cas(Y410) phosphorylation activated by EGF and ERK general phosphorylation activated by Fn, also decreased the invasiveness and proliferation of SGC7901 cells activated by EGF or Fn.

Conclusion: There is crosstalk between EGFR and integrin signal transduction. FAK may be a key cross point of the two signal pathways and acts as a potential target for human gastric cancer therapy.

Keywords: gastric adenocarcinoma, epidermal growth factor receptor, integrin, focal adhesion kinase, crosstalk

\section{Introduction}

Gastric cancer remains a major public health issue as the fourth most common cancer and the second leading cause of cancer death worldwide. ${ }^{1,2}$ Conventional therapies for gastric cancer include resection and chemotherapy, but these measures are noncurative for those patients who are diagnosed with advanced gastric cancer. As a result, new therapeutic paradigms and alternative therapies options are needed urgently for more effective treatment of this aggressive malignancy. Molecular-targeted therapy is an emerging technology that shows considerable promise as a novel treatment option. Many drugs used for molecular-targeted therapy have been approved by the US Food and Drug Administration, some of which have been proved to be impressively effective. ${ }^{3-5}$ For example, imatinib is now considered the first-line therapy in treating chronic myeloid leukemia. ${ }^{5}$ This strategy is based on the observation of abnormal molecular signal transductions in tumor cells. However, there are crosstalks among signal transductions that have weaved different 
signal pathways into a complicated network. When a signal molecule binds its ligand, different reactions downstream of the pathway are often observed in different tissues or species because of the complexity of the signal network, which requires further challenging research to elucidate. ${ }^{6}$ In the present study, we tested crosstalk between downstream pathways of integrin and epidermal growth factor receptor (EGFR). We also aimed for a cross point, focal adhesion kinase (FAK), blocked its synthesis by short-interfering RNA (siRNA), examined the effect of crosstalk on the invasion and proliferation of human gastric adenocarcinoma cells. This study represented the first evidence of a crosstalk between EGFR and integrin signal pathways in gastric adenocarcinoma cell lines, and proved FAK to be a cross point of two signal pathways, which makes it a potent molecular target for gastric adenocarcinoma therapy.

\section{Methods}

\section{Materials}

Fibronectin (Fn) and epidermal growth factor (EGF) were bought from Sigma-Aldrich (St Louis, MO). RPMI-1640 and fetal bovine serum were purchased from Gibco (Carlsbad, CA). Oligofectamine and Plus Reagent were obtained from Invitrogen (Carlsbad, CA). Rabbit anti-human FAK, FAK(Y397) phosphorylation, rabbit anti-human p130cas, rabbit antihuman p130cas(Y410) phosphorylation, rabbit anti-human ERK (ERK1 + ERK2), and rabbit anti-human ERK general phosphorylation (T185 + Y187 + T202 + Y204) antibodies were purchased from Abcam (Cambridge, MA). Boyden chambers (Millicell Chamber) were obtained from Millipore (Billerica, MA). Matrigel was obtained from BD Biosciences (Franklin Lakes, NJ). Double-stranded siRNAs were synthesized by Shenggong Biotechnology (Shanghai, China). Silencer ${ }^{\mathrm{TM}}$ negative control siRNA was brought from Ambion (Austin, TX). Electrochemiluminescence kit was bought from Boshide (Wuhan, China). Other reagents were products of Sigma-Aldrich. The human gastric adenocarcinoma cell line, SGC7901, was obtained from China Centre for Type Culture Collection (Shanghai, China). A stock culture of SGC7901 was maintained at $37^{\circ} \mathrm{C}$ in RPMI-1640 medium, supplemented with $10 \%$ fetal bovine serum in humidified atmosphere containing $5 \% \mathrm{CO}_{2}$ and $95 \%$ air.

\section{The effect of crosstalk between EGFR and integrin on expression and phosphorylation of downstream molecules in both signal pathways}

Immunoprecipitation and western blot assays were used to measure expression and phosphorylation of downstream molecules in both EGFR and integrin pathways. SGC7901 cells were grown to a confluence of $1.5 \times 107$ cells $/ \mathrm{cm}^{2}$ in RPMI-1640 medium containing $10 \%$ fetal bovine serum. Before the experiments, the SGC7901 cells were cultured overnight in RPMI1640 medium without fetal bovine serum. Fn and EGF were added into the medium with a final concentration of $10 \mu \mathrm{g} / \mathrm{L}$ and $100 \mu \mathrm{g} / \mathrm{L}$, respectively. After 10 minutes, the medium was removed and cells were pelleted, lyzed in ice-cold lysis buffer (Tris-HCl, $25 \mathrm{mmol} / \mathrm{L}$; edetic acid, $1 \mathrm{mmol} / \mathrm{L}$; $\mathrm{NaCl}, 150 \mathrm{mmol} / \mathrm{L}$; NaF, $50 \mathrm{mmol} / \mathrm{L}$; Triton-100, 1\%; $\mathrm{PMSF}, 1 \mathrm{mmol} / \mathrm{L}$, leupeptin, $1 \mathrm{mg} / \mathrm{L}$; aprotinin, $1 \mu \mathrm{mol} / \mathrm{L}$; $\mathrm{pH}, 7.6)$. Each sample was centrifuged at $100 \mathrm{~g} /$ minute for 10 minutes. The precipitation was resuspended and incubated on ice for 30 minutes, then centrifuged at $10,000 \mathrm{~g} /$ minute for 15 minutes at $4^{\circ} \mathrm{C}$. Rabbit anti-FAK, p130cas, and ERK antibodies were added into the supernatant, respectively. After incubating at $4^{\circ} \mathrm{C}$ for 1 hour, $50 \mu \mathrm{L}$ protein $\mathrm{G}$ bead slurry was added into each sample. The samples were rotated for 1 hour at $4^{\circ} \mathrm{C}$, centrifuged at $10,000 \mathrm{~g} /$ minute for 30 seconds at $4^{\circ} \mathrm{C}$. The supernatant was removed. Beads were washed five times and mixed with laemmli buffer, heated at $90^{\circ} \mathrm{C}$ for 10 minutes and centrifuged at 10,000 g/minutes for 5 minutes. Equivalent amounts of protein were separated by sodium dodecyl sulfate - polyacrylamide gel electrophoresis (SDS-PAGE). Protein on the SDS-PAGE was transferred to nitrocellulose using a semidry transfer apparatus (Bio-Rad, Berkeley, CA), blocked with $3 \%$ bovine serum albumin in TBST (Tris, $10 \mathrm{mmol} / \mathrm{L} ; \mathrm{pH}, 8.0 ; \mathrm{NaCl}, 150 \mathrm{mmol} / \mathrm{L}$; Tween 20, $0.1 \%$ ), then added to rabbit anti-human FAK, FAK(Y397) phosphorylation, rabbit anti-human p130cas, rabbit antihuman p130cas(Y410) phosphorylation, rabbit anti-human ERK, rabbit anti-human ERK general phosphorylation antibodies, then rotated for 1 hour at room temperature and washed with TBST three times. The proteins on the nitrocellulose were detected by an electrochemiluminescence kit (Boshide) including a goat anti-rabbit antibody according to the manufacturer's instructions.

\section{Effect of siRNA targeting FAK on crosstalk between EGFR and integrin}

The siRNA sequence targeting human FAK was GUGUGCUCUUGGUUCAAGCTT, designed with the software developed by Ambion Inc according to a messenger RNA sequence of FAK in GenBank (Bethesda, MD). The FAK siRNA was transfected into SGC7901 cells with lipofectamine 2000 according to the manufacturer's protocol. 
Silencer ${ }^{\mathrm{TM}}$ negative-control siRNA was used as negative control. SGC7901 transfected with FAK siRNA was named SGC7901/FAKBLOCK. After transfection for 36 hours, cells of SGC7901/FAKBLOCK were stimulated with $10 \mu \mathrm{g} / \mathrm{L}$ Fn and $100 \mu \mathrm{g} / \mathrm{L}$ EGF for 10 minutes, respectively. Then, total cellular extracts were prepared, expression and phosphorylation of FAK, p130cas, and ERK were tested by immunoprecipitation and western blotting as described previously.

\section{The effect of siRNA targeting FAK on invasion and proliferation of SGC790 I}

Invasion assays were performed with modified Boyden chamber assay. The filter membrane on the bottom of each millicell chamber was coated with human matrigel. About $1 \times 10^{5}$ cells of SGC7901 or SGC7901/FAKBLOCK were added into each millicell chamber, five chambers per group. Fn and EGF were added into the chambers with final concentrations of $10 \mu \mathrm{g} / \mathrm{L}$ and $100 \mu \mathrm{g} / \mathrm{L}$, respectively. The chambers were put into 24 -well cell culture plates, maintained at $37^{\circ} \mathrm{C}$ in humidified atmosphere containing $5 \% \mathrm{CO}_{2}$ and $95 \%$ air for 12 hours. The cells on the lower surfaces of filter membranes were dyed with routine hematoxylin and eosin, and counted. Results from quintuple wells were expressed as means \pm standard deviation.

A proliferation assay was performed with MTT assay. SGC7901 or SGC7901/FAKBLOCK cells were seeded in a 96-well plate at a density of $1 \times 10^{4}$ cells per well. The medium was replaced with fresh medium containing Fn and EGF with final concentrations of $10 \mu \mathrm{g} / \mathrm{L}$ and $100 \mu \mathrm{g} / \mathrm{L}$, respectively. Cells were cultured in an incubator at $37^{\circ} \mathrm{C}$, containing $5 \% \mathrm{CO}_{2}$. Twenty microliters of MTT reagent (prepared to $5 \mathrm{mg} / \mathrm{mL}$ ) was added into each well at 20 hours, 4 hours prior to completion of the incubation periods. The mixture was carefully taken out and $150 \mu \mathrm{L}$ of dimethyl sulfoxide was added into each well by pipetting up and down several times unless the content homogenized. The plates were kept on a rocker shaker for 10 minutes at room temperature, and read at $595 \mathrm{~nm}$ with a microplate reader.

\section{Statistical analysis}

Results were compared using analysis of variance (ANOVA) and Student's $t$-test and considered statistically significant when $P<0.05$. Calculations were done using SPSS for Windows (version 11.5; IBM, Armonk, NY). All experiments were done independently at least three times unless indicated otherwise.

\section{Results}

The effect of cross talk between EGFR and integrin on expression and phosphorylation of downstream molecules in both signal pathways

Neither EGF nor Fn could increase the expression of ERK, FAK, and p130cas (Figure 1). However, the expression of FAK in SGC7901/FAKBLOCK decreased 70\%, compared with SGC7901. There was no difference between SGC7901 and SGC7901 transfected with Silencer ${ }^{\mathrm{TM}}$ negative control siRNA (data not shown).

In SGC7901 cells, ERK general phosphorylation increased 2.90 times after being stimulated by Fn, and 4.38 times by EGF (Figure 2). FAK(Y397) phosphorylation increased 2.75 times after being stimulated by EGF, and 5.00 times by Fn (Figure 3). p130cas(Y410) phosphorylation increased 4.33 times after being stimulated by EGF, and 6.67 times by Fn (Figure 4).

\section{Effect of siRNA targeting FAK on cross talk between EGFR and integrin}

The FAK(Y397) phosphorylation in SGC7901/FAKBLOCK was only $32 \%$ of that in SGC7901 after being stimulated by EGF, and $27 \%$ of that in SGC7901 by Fn (Figure 3 ). ERK general phosphorylation in SGC7901/FAKBLOCK

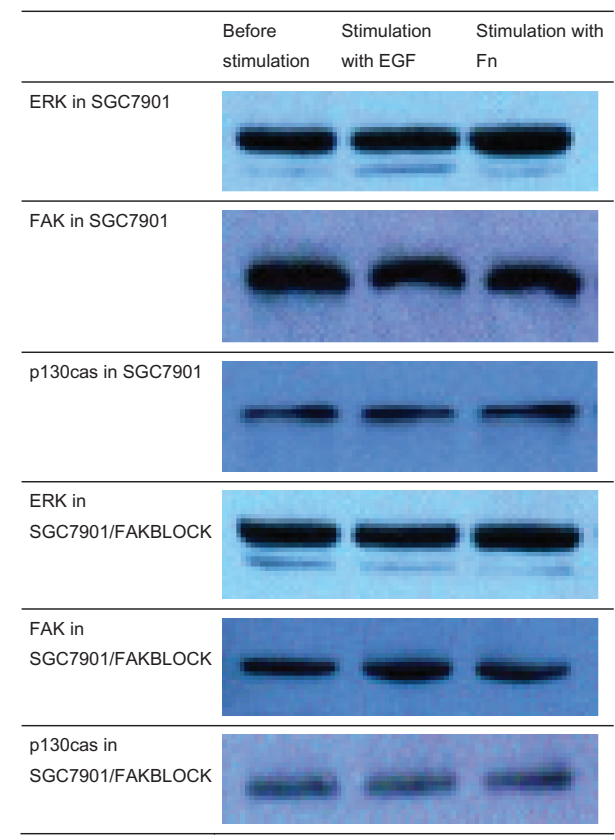

Figure I Expression of ERK, FAK, and pI30cas with stimulation of EGF or Fn. Notes: There was no difference between expression of ERK, FAK, and pI 30 cas before and after stimulation with EGF or Fn $(P>0.05, \bar{x} \pm \mathrm{s} n=3)$. Expression of FAK in SGC790 I/FAKBLOCK was only $30 \%$ of that in SGC790I $(P<0.05, \bar{x} \pm s, n=3)$. Abbreviations: ERK, extracellular signal-regulated kinase; FAK, focal adhesion kinase; EGF, epidermal growth factor; Fn, fibronectin. 
A

\begin{tabular}{llll}
\hline & $\begin{array}{l}\text { Before } \\
\text { stimulation }\end{array}$ & $\begin{array}{l}\text { Stimulation } \\
\text { with EGF }\end{array}$ & $\begin{array}{l}\text { Stimulation with } \\
\text { Fn }\end{array}$ \\
\hline $\begin{array}{l}\text { ERK general } \\
\text { phosphorylation in }\end{array}$ & & \\
SGC7901 & & \\
\hline ERK general & & \\
phosphorylation in & & \\
SGC7901/FAKBLOCK & & & \\
\hline
\end{tabular}

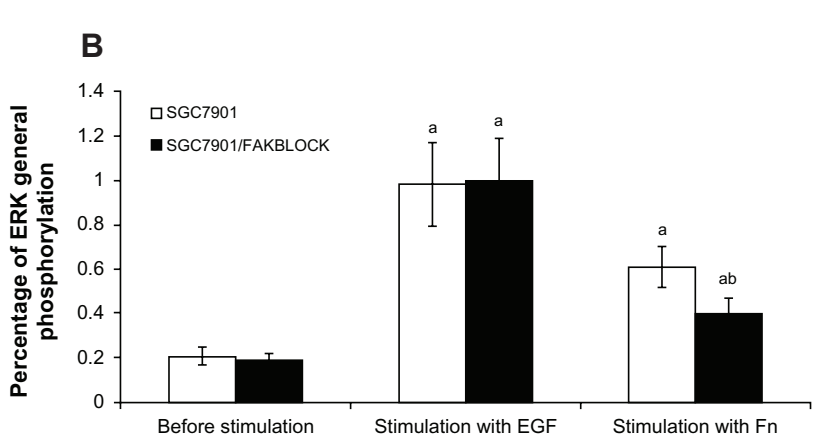

Figure 2 (A) ERK general phosphorylation in SGC790I and SGC790I/FAKBLOCK with stimulation of EGF or Fn respectively. (B) Digital analysis of western blot $(\bar{x} \pm \mathrm{s}, \mathrm{n}=3)$.

Notes: (a) Compared with ERK general phosphorylation before stimulation, $P<0.05$; (b) Compared with ERK general phosphorylation in SGC790I after stimulation with $\mathrm{Fn}, P<0.05$. Maximum phosphorylation is defined as $100 \%$.

Abbreviations: ERK, extracellular signal-regulated kinase; EGF, epidermal growth factor; Fn, fibronectin.

A

\begin{tabular}{llll}
\hline & $\begin{array}{l}\text { Before } \\
\text { stimulation }\end{array}$ & $\begin{array}{l}\text { Stimulation } \\
\text { with EGF }\end{array}$ & $\begin{array}{l}\text { Stimulation with } \\
\text { Fn }\end{array}$ \\
\hline FAK Y397 & & \\
phosphorylation in & & \\
SGC7901 & & \\
\hline FAK Y397 & & \\
phosphorylation in \\
SGC7901/FAKBLOCK
\end{tabular}

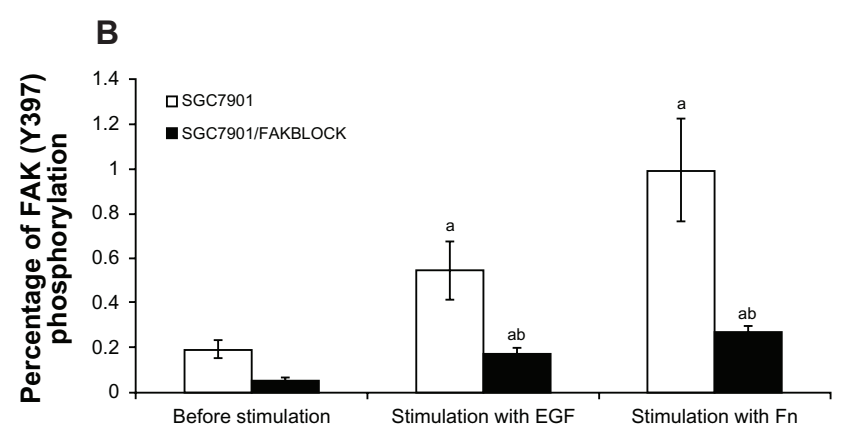

Figure 3 (A) FAK(Y397) phosphorylation in SGC790I and SGC790I/FAKBLOCK with stimulation of EGF or Fn. (B) Digital analysis of western blot $(\bar{x} \pm s, n=3)$. Notes: (a) Compared with FAK(Y397) phosphorylation before stimulation, $P<0.05$; (b) Compared with FAK(Y397) phosphorylation after stimulation in SGC790I, $P<0.05$. Maximum phosphorylation is defined as $100 \%$.

Abbreviations: FAK, focal adhesion kinase; EGF, epidermal growth factor; Fn, fibronectin.
A

\begin{tabular}{llll}
\hline & $\begin{array}{l}\text { Before } \\
\text { stimulation }\end{array}$ & $\begin{array}{l}\text { Stimulation } \\
\text { with EGF }\end{array}$ & $\begin{array}{l}\text { Stimulation with } \\
\mathrm{Fn}\end{array}$ \\
\hline p130cas Y410 & & & \\
phosphorylation in & & & \\
SGC7901 & & & \\
\hline p130cas Y410 & & \\
phosphorylation in & & & \\
SGC7901/FAKBLOCK & & & \\
\hline
\end{tabular}

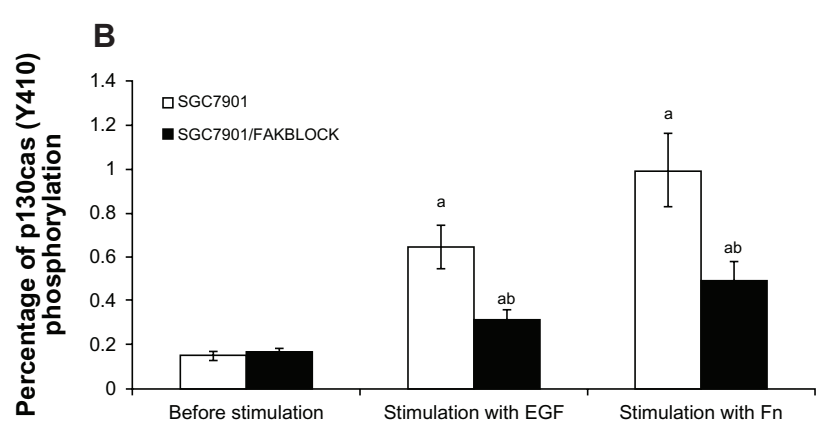

Figure 4 (A) pl30cas(Y4I0) phosphorylation in SGC790I and SGC790I/ FAKBLOCK with stimulation of EGF or Fn respectively. (B) Digital analysis of western blot $(\bar{x} \pm \mathrm{s}, \mathrm{n}=3)$.

Notes: (a) Compared with pl30cas(Y4I0) phosphorylation before stimulation, $P<0.05$; (b) Compared with pl30cas(Y4I0) phosphorylation in SGC790I after stimulation with EGF or Fn, $P<0.05$. Maximum phosphorylation is defined as $100 \%$. Abbreviatons: EGF, epidermal growth factor; Fn, fibronectin.

increased after being stimulated by Fn, but was only $66 \%$ of that in SGC7901 (Figure 2). p130cas(Y410) phosphorylation also increased after being stimulated by EGF or Fn in both SGC7901 and SGC7901/FAKBLOCK, but the p130cas(Y410) phosphorylation in SGC7901/FAKBLOCK was only $49 \%$ of that in SGC7901 by stimulation of EGF (Figure 4).

\section{The effect of siRNA targeting FAK on invasion and proliferation of SGC790 I}

A variety of in vitro test systems has been used to assess the cell's ability to cross tissue barriers. The basement membranes in the tissues have been assumed to create a critical barrier to the passage of cells. The modified Boyden chambers use human matrigel as a coating on the top of a filter as a barrier to the passage of cells, which is considered a convenient and objective way to measure invasiveness of different tumor cells. The SGC7901 cells on the lower bottom of Boyden chambers increased 1.50 times after being stimulated by EGF, and 2.36 times by Fn. SGC7901/FAKBLOCK cells on the lower bottom of Boyden chambers also increased significantly after being stimulated by EGF, but were only $48 \%$ of SGC7901; and also increased significantly after stimulation of Fn, but were only $37 \%$ of SGC7901. There was statistical difference between SGC7901 and SGC7901/FAKBLOCK 
cells on lower bottom of Boyden chambers after stimulation with EGF or Fn (Figure 5).

The MTT assays are colorimetric assays with a main application which allows viability to be assessed and proliferation of cells. MTT value of both SGC7901 and SGC7901/ FAKBLOCK cells at 24 hours was much higher with stimulation of EGF or Fn than the controls. MTT value of SGC7901/ FAKBLOCK was less than SGC7901 with stimulation by EGF or Fn. The SGC7901/FAKBLOCK MTT value was only $77 \%$ of SGC7901 at 24 hours by EGF, and $63 \%$ by stimulation of Fn, which had statistically significant differences (Figure 6).

\section{Discussion}

Recently some researchers announced that moleculartargeted drugs are too simple for common cancers and hitting a single target is unlikely to halt tumor progression in complicated cancers such as prostate and colon cancers.? Based on a thorough analysis of current available research findings, a conclusion can be drawn that this phenomenon may be induced by crosstalks among signal pathways. In the network of signal transduction, crosstalks form branches and shunt the signals from the trunk when they are blocked by

A

Before stimulation Stimulation with EGF Stimulation with Fn
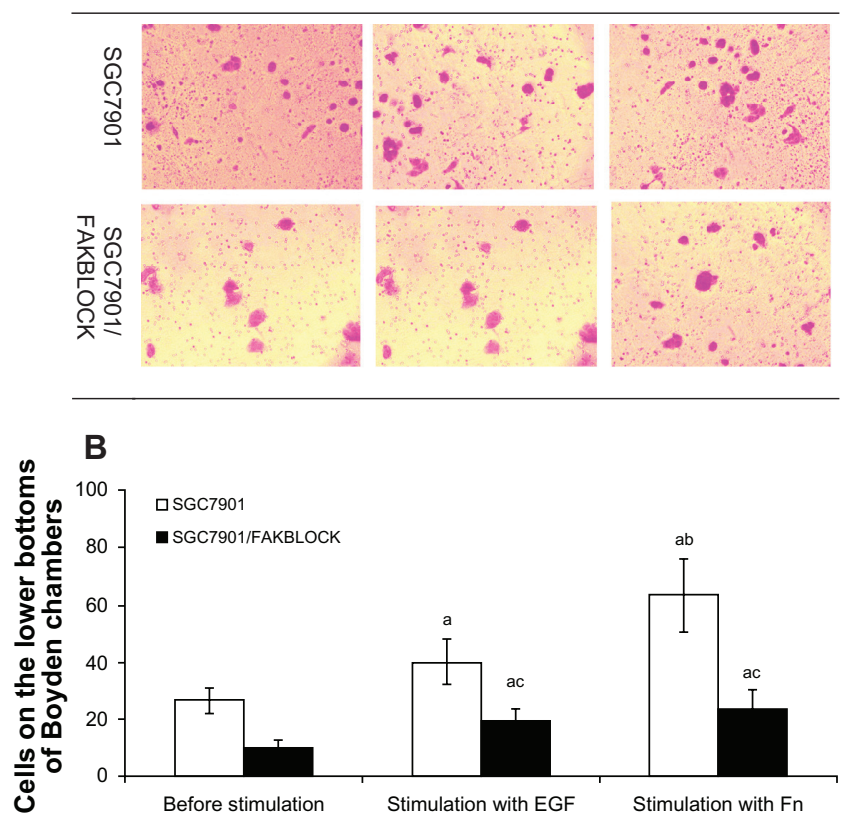

Figure 5 (A) Cells of SGC790I and SGC790I/FAKBLOCK on the lower surfaces of filter membranes in Boyden chambers with stimulation of EGF or Fn. (B) Analysis of invasiveness of SGC790 I and SGC790I/FAKBLOCK with stimulation of EGF or Fn $(\bar{x} \pm \mathrm{s}, \mathrm{n}=5)$.

Notes: (a) Compared with invasiveness before stimulation, $P<0.05$; (b) Compared with invasiveness after stimulation with EGF, $P<0.05$; (c) Compared with SGC790 I after stimulation.

Abbreviations: EGF, epidermal growth factor; Fn, fibronectin.

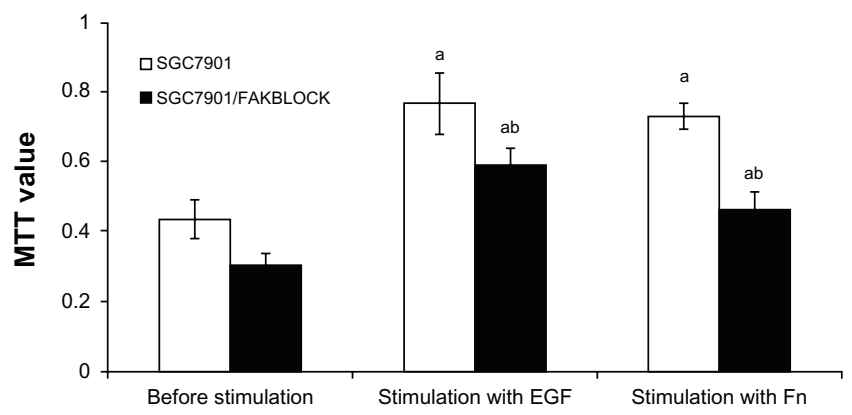

Figure 6 Analysis of MTT value of SGC790I and SGC790I/FAKBLOCK after stimulation with EGF or Fn $(\bar{x} \pm s, n=5)$.

Notes: (a) Compared with MTT value before stimulation, $P<0.05$; (b) Compared with SGC790I after stimulation, $P<0.05$.

molecular-targeted drugs. Here we showed there is a crosstalk between EGFR and integrin signal pathways in gastric adenocarcinoma, and attempted to reveal the complexity of the crosstalk, which may be responsible for unavailability of some molecular-targeted drugs in certain cancers.

Classic downstream pathways of the EGFR signal pathway are Ras/Raf/MEK/ERK/MAPK and PI3K/PDK1/Akt (protein kinase $\mathrm{B}[\mathrm{PKB}]),{ }^{8,9}$ and the downstream pathway of integrin is FAK/paxillin and p130cas. ${ }^{10-13}$ Crosstalks of the two signal pathways are hot issues for many researchers ${ }^{14}$, since both EGFR and integrin pathways are important for tumor cell invasion and proliferation. If there are crosstalks between EGFR and integrin pathways, it must be difficult or impossible for a single molecular-targeted drug to block all biological functions such as proliferation and growth induced by EGFR or integrin pathways in cancer cells. Unfortunately, it has been proved that there is a crosstalk between EGFR and integrin $\alpha v \beta 5$ in human pancreatic carcinoma, which involves tumor cell invasion and proliferation. ${ }^{14,15} \mathrm{We}$ conjectured that there are also crosstalks between EGFR and integrin pathways in human gastric adenocarcinoma. Once EGF binds its ligand, EGFR, activated signals include not only ERK and PKB, but also FAK, paxillin, and p130cas, which are thought to be downstream of integrin. Under such circumstances, it is not enough to block EGFR or the integrin signal pathway alone to inhibit proliferation and invasion of human gastric adenocarcinoma cells. A better way is to block both signal pathways or inhibit the cross-point that links the two signal pathways, EGFR and integrin.

In this experiment, there was a rapid increase of phosphorylation in FAK(Y397) and p130cas(Y410) after stimulation with Fn, so was general phosphorylation of ERK with EGF. Both EGF and Fn stimulated proliferation and invasion of the gastric adenocarcinoma cell line, SGC7901. These data are not surprising, because similar results have been reported 
previously. ${ }^{8-13}$ Here, we stress our new finding in this experiment that EGF caused tyrosine phosphorylation of FAK(Y397) and p130cas(Y410), and Fn caused general phosphorylation of ERK, which were neglected before. These discoveries suggested there is a crosstalk between EGFR and integrin signal pathways in gastric adenocarcinoma cells.

Once there is crosstalk, the cross point will be an attractive focus. Blockage of the cross point will affect downstream signal transduction in both signal pathways, as an intersection of different branches is blocked. FAK is a key molecule downstream of integrin. Recent researchers found EGFRvIII-promoted glioma cell migration was closely linked to high levels of tyrosine phosphorylation in FAK Y397. ${ }^{16}$ EGFR and FAK may be bridged by a PAK-phosphorylated alternate-spliced isoform of the steroid receptor coactivator- $3 .{ }^{17}$ Based on these data, we assumed that FAK may be the cross point of EGFR and integrin pathways, and designed siRNA of FAK to block synthesis of FAK to see whether decreasing of FAK expression could affect phosphorylation of downstream molecules in both signal pathways, and eventually influence the biological functions of gastric adenocarcinoma cells. As mentioned in the results, after FAK siRNA was transfected into SGC7901, the quantity of FAK protein was decreased to only $30 \%$ of the controls, and this subpopulation was named SGC7901/FAKBLOCK. In SGC7901/FAKBLOCK, not only phosphorylation of FAK(Y397), but also invasion and proliferation of the cells are decreased. The phosphorylation of p130cas(Y410) in SGC7901/FAKBLOCK was evidently weaker than that in SGC7901 after being stimulated by Fn. This proved FAK is a key molecule in the integrin pathway. In the meantime, the general phosphorylation of ERK in SGC7901/FAKBLOCK was also weaker than that in SGC7901 after stimulation with Fn, which means the integrin pathway affects EGFR signal downstream transduction through FAK. The p130cas(Y410) phosphorylation in SGC7901/BLOCK was also weaker than that in SGC7901 after stimulation with EGF, which indicates the EGFR pathway activates integrin downstream signal molecules through FAK.

\section{Conclusion}

In summary, we proved there is a crosstalk between EGFR and integrin pathways, which reduces effects of moleculartargeted drugs targeted to both signal pathways. FAK is the cross point of the two signal pathways. Blockage of FAK can decrease the crosstalk between EGFR and integrin pathways, and inhibit proliferation and invasion of gastric adenocarcinoma cell line, SGC7901. FAK may be a more effective target than other molecules in the two signal pathways.
These results may provide new ideas for gastric cancer treatment with molecular-targeted therapy.

\section{Acknowledgments}

This work was supported by grants from Natural Science Foundation of Fujian Province, China (Grant number: 2009J01148), Innovation Fund of Fujian Provincial Department of Health, China (Grant number: 2009-CXB-14) and Nursery Research Fund of Fujian Medical University, China (Grant number: 2010MP033).

\section{Disclosure}

The authors report no conflicts of interest in this work.

\section{References}

1. Crew KD, Neugut AI. Epidemiology of gastric cancer. World $J$ Gastroenterol. 2006;12(3):354-362.

2. Ferlay J, Shin HR, Bray F, et al. Estimates of worldwide burden of cancer in 2008: GLOBOCAN 2008. Int J Cancer. 2010;127(12):2893-2917.

3. Hida T, Ogawa S, Park JC, et al. Gefitinib for the treatment of nonsmall-cell lung cancer. Expert Rev Anticancer Ther. 2009;9(1):17-35.

4. Boysen M, Longson C, Stevens A. Erlotinib for the treatment of nonsmall-cell lung cancer. Lancet Oncol. 2009;10(1):15-16.

5. Kantarjian HM, Larson RA, Guilhot F, et al. Efficacy of imatinib dose escalation in patients with chronic myeloid leukemia in chronic phase. Cancer. 2009;115(3):551-560.

6. Dragovich T, McCoy S, Fenoglio-Preiser CM, et al. Phase II trial of erlotinib in gastroesophageal junction and gastric adenocarcinomas: SWOG 0127. J Clin Oncol. 2006;24(30):4922-4927.

7. Coombes R. Molecular targeted drugs are too simple for common cancers, says UK researcher. BMJ. 2010;340:c153.

8. Avraham R, Yarden Y. Feedback regulation of EGFR signalling: decision making by early and delayed loops. Nat Rev Mol Cell Biol. 2011;12(2):104-117.

9. Bazley LA, Gullick WJ. The epidermal growth factor receptor family. Endocr Relat Cancer. 2005;12:S17-S27.

10. Yun SP, Ryu JM, Han HJ. Involvement of $\beta 1$-integrin via PIP complex and FAK/paxillin in dexamethasone-induced human mesenchymal stem cells migration. J Cell Physiol. 2011;226(3):683-692.

11. Nalla AK, Asuthkar S, Bhoopathi P, et al. Suppression of uPAR retards radiation-induced invasion and migration mediated by integrin $\beta 1 / \mathrm{FAK}$ signaling in medulloblastoma. PLoS One. 2010;5(9):e13006.

12. Li D, Ding J, Wang X, et al. Fibronectin promotes tyrosine phosphorylation of paxillin and cell invasiveness in the gastric cancer cell line AGS. Tumori. 2009;95(6):769-779.

13. Zouq NK, Keeble JA, Lindsay J, et al. FAK engages multiple pathways to maintain survival of fibroblasts and epithelia: differential roles for paxillin and p130Cas. J Cell Sci. 2009;122(pt3):357-367.

14. Ricono JM, Huang M, Barnes LA, et al. Specific cross talk between epidermal growth factor receptor and integrin alphavbeta5 promotes carcinoma cell invasion and metastasis. Cancer Res. 2009;69(4):1383-1391.

15. Kim SH, Kim SH. Antagonistic effect of EGF on FAK phosphorylation/ dephosphorylation in a cell. Cell Biochem Funct. 2008;26(5): 539-547.

16. Liu M, Yang Y, Wang C, et al. The effect of epidermal growth factor receptor variant III on glioma cell migration by stimulating ERK phosphorylation through the focal adhesion kinase signaling pathway. Arch Biochem Biophys. 2010;502(2):89-95.

17. Tomar A, Schlaepfer DD. A PAK-activated linker for EGFR and FAK. Dev Cell. 2010;18(2):170-172. 
OncoTargets and Therapy

\section{Publish your work in this journal}

OncoTargets and Therapy is an international, peer-reviewed, open access journal focusing on the pathological basis of all cancers, potential targets for therapy and treatment protocols employed to improve the management of cancer patients. The journal also focuses on the impact of management programs and new therapeutic agents and protocols on
Dovepress

patient perspectives such as quality of life, adherence and satisfaction. The manuscript management system is completely online and includes a very quick and fair peer-review system, which is all easy to use. Visit http://www.dovepress.com/testimonials.php to read real quotes from published authors.

\footnotetext{
Submit your manuscript here: http://www.dovepress.com/oncotargets-and-therapy-journal
} 